\title{
Etiologies of the Relationships Among Body Mass Index and Cold-Heat Patterns: A Twin Study
}

\author{
Yoon-Mi Hur, ${ }^{1}$ Hee-Jeong Jin, ${ }^{2}$ and Siwoo Lee ${ }^{2}$ \\ ${ }^{1}$ Research Institute for Welfare Society, Mokpo National University, Jeonnam, South Korea \\ ${ }^{2}$ Mibyeong Research Center, Korea Institute of Oriental Medicine, Daejeon, South Korea
}

\begin{abstract}
The phenotypic relationships between body mass index (BMI) and cold-heat patterns have been frequently reported, but the etiology of these relationships remains unknown. We previously demonstrated that the cold pattern (CP) and the heat pattern (HP) were heritable traits. In the present study, we explored underlying genetic and environmental structures of the relationships among BMI and the CP and the HP. Twins $(N=1,752)$ drawn from the South Korean twin registry completed a cold-heat pattern questionnaire via a telephone interview. The phenotypic correlations among the three phenotypes were moderate but significant. Cross-twin, cross-trait correlations among BMI and the CP and the HP were consistently greater in monozygotic than in dizygotic twins, suggesting the presence of genetic effects on the relationships between $\mathrm{BMI}$ and the two patterns. A trivariate Cholesky model was applied to the raw data. The results indicated that the phenotypic relationship between the HP and BMI was completely determined by common genetic influences, while the relationship between the $\mathrm{CP}$ and $\mathrm{BMI}$ was explained by both common genetic and common individual-specific environmental influences. The genetic correlation between the HP and the $\mathrm{CP}$ was not significant, suggesting that the two patterns may be genetically independent from each other. Genetic correlations were 0.31 between the HP and BMI, and -0.22 between the CP and BMI. The individual-specific environmental correlation was -0.22 between $\mathrm{HP}$ and $\mathrm{CP}$, and between $\mathrm{CP}$ and $\mathrm{BMI}$.
\end{abstract}

Keywords: cold and heat patterns, body mass index, twin, gene, environment

In East Asian medicine, the cold-heat pattern differentiation system has long been used in clinical diagnosis as well as in the treatment of patients suffering from various diseases, including menopausal disorders, chronic rhinitis, dyspepsia, diarrhea, dysmenorrhea, headache, inflammation in the digestive tract, atopic dermatitis (Bae et al., 2017), and rheumatoid arthritis (Ferreira \& Lopes, 2011). The cold pattern (CP) is characterized by a preference for warm temperature, intolerance of cold (high cold susceptibility), hypothermia, paleness, diarrhea, peripheral chills, and spasms, whereas the heat pattern (HP) is characterized by diaphoresis, rapid pulse, flushed face, vexation, constipation, thirst, aversion to hot temperature (high heat susceptibility), and deep-colored urine (Jian, 2005). It has been suggested that incorporation of cold-heat patterns in diagnosis gives clinicians information about the nature and the location of physiological imbalances of the patient, which can improve diagnostic accuracy and the efficacy of the treatment (WHO Regional Office for the Western Pacific, 2007).
Several studies have consistently shown that body mass index (BMI) is positively correlated with the HP and negatively correlated with the CP (Anderson, 1999; Lee et al., 2018; Mun et al., 2017; Pham et al., 2016). However, shared etiologies of these three traits remain unknown. Twin studies have demonstrated that BMI is highly heritable (Hur et al., 2008; Silventoinen et al., 2016). In support of the findings from twin studies, gene identification studies have successfully detected several FTO gene variants related to BMI (e.g., Qi et al., 2014). Using the sample employed in the present study, we recently showed that the $\mathrm{CP}$ and the HP are heritable traits, with heritability estimates being $40 \%(95 \%$ CI $[38,42])$ for the former and 33\% (95\% CI

RECEIVED 2 February 2018; ACCEPTED 26 February 2018. First published online 30 April 2018.

ADDRESS FOR CORRESPONDENCE: Siwoo Lee, Mibyeong Research Center, Korea Institute of Oriental Medicine, Daejeon, South Korea. E-mail: bfree@kiom.re.kr 
$[25,42)$ for the latter (Hur et al., 2018). As with many other complex traits, shared environmental influences on the $\mathrm{CP}$ and the HP were negligible, and environmental factors that influenced the $\mathrm{CP}$ and the HP were mainly individual specific. These results suggest that cold-heat patterns are complex, multi-factorial phenotypes caused by a combination of genetic and environmental factors. In the present study, we investigated whether the relationships between cold-heat patterns and BMI arise due to shared genetic and/or environmental etiology.

\section{Material and Methods}

\section{Sample}

The sample consisted of 1,752 (1,047 monozygotic (MZ) and 705 dizygotic $(D Z)$ ) twins (mean age $=19.1 \mathrm{yrs} ; S D$ of age $=3.1 \mathrm{yrs}$; range $=12-29 \mathrm{yrs}$ ) drawn from the South Korean Twin Registry (Hur et al., 2013). A detailed description of the present sample can be found in Hur et al. (2013, 2018).

three-item zygosity questionnaire was used to determine twins' zygosity (Ooki et al., 1993). When compared to DNA analysis, this approach has been shown to achieve over $90 \%$ accuracy (Ooki et al., 1993). The mean (SD) of BMI in our sample was 21.3 (3.1), which was very close to that of adolescents and young adults in South Korea (Park, 2011), suggesting that our sample is fairly representative of South Korean adolescents and young adults in terms of BMI. Means and standard deviations of the $\mathrm{CP}$ and the $\mathrm{HP}$ in our sample were reported elsewhere (Hur et al., 2018).

\section{Measures}

A telephone interview was undertaken with twins to complete a questionnaire of cold-heat patterns (Yeo et al., 2016) and report their current height and weight. BMI was calculated as weight $(\mathrm{kg})$ divided by height $\left(\mathrm{m}^{2}\right)$. The cold-heat patterns questionnaire used in the present study was comprised of eight items for typical symptoms of the CP and seven items for typical symptoms of the HP (see Table 2 in Hur et al., 2018 for the items). Twins were instructed to respond to five-point Likert scale $(1=$ not at all true, $5=$ very true) for each item. Responses to the HP and the $\mathrm{CP}$ items were summed so that higher scores on the HP indicate higher $\mathrm{HP}$ and higher scores on the $\mathrm{CP}$ indicate higher CP. This questionnaire was developed for the general population through factor analysis and clinicians' diagnostic consensus. The items in the questionnaire have been demonstrated to have adequate psychometric properties (Yeo et al., 2016). Cronbach alpha reliabilities in the present sample were 0.66 for the $\mathrm{CP}$ and 0.70 for the HP.

\section{Statistical Analysis}

To determine the causes of covariance among BMI, the $\mathrm{HP}$, and the CP, twin correlations and cross-twin, crosstrait correlations for $\mathrm{MZ}$ and $\mathrm{DZ}$ twins were computed and

\section{TABLE 1}

Age- and Sex-Corrected Phenotypic Correlations (Below the Diagonal) and MZ and DZ Cross-Twin, Cross-Trait Correlations (Above the Diagonal) for the Cold Pattern (CP), the Heat Pattern (HP), and body mass index (BMI).

\begin{tabular}{lclc}
\hline & CP & HP & BMI \\
\hline CP & - & $-0.07(0.03)$ & $-0.18^{* *}(0.04)$ \\
HP & $-0.16^{* *}$ & - & $0.18^{* *}(0.03)$ \\
BMI & $-0.20^{* *}$ & $0.19^{* *}$ & - \\
\hline
\end{tabular}

Note: Cross-twin, cross-trait correlations for DZ twins are in parenthesis. ${ }^{* *} p<0.01$.

model-fitting analyses were carried out. As our previous study has shown no significant sex or age differences in genetic and environmental influences on the HP and the CP (Hur et al., 2018), prior to twin correlation and modelfitting analyses, we combined both sexes and corrected the data for age, age ${ }^{2}$, sex, and age $\times$ sex, using a regression method (McGue \& Bouchard, 1984).

A trivariate Cholesky model (Neale \& Cardon, 1992) was applied to the data. In the full trivariate Cholesky model, each of the three Cholesky factors for the CP, the HP, and $\mathrm{BMI}$ are decomposed into additive genetic factors $(\mathrm{A}$, the sum of the average effect of all alleles that influence a phenotype), shared environmental factors ( $\mathrm{C}$, those environmental factors shared between the two members of a twin pair) and individual specific environmental factors ( $\mathrm{E}$, those environmental factors specific to each member of a twin pair and measurement error). The first Cholesky factors (i.e., $\mathrm{A} 1, \mathrm{C} 1$, and E1) exert influences on all three traits, that is, the $\mathrm{CP}$, the HP, and BMI, although they mainly influence the CP. The second Cholesky factors (i.e., A2, C2, and E2) have effects mainly on the HP, although they also influence BMI. The third Cholesky factors (i.e., A3, C3, and E3) are those unique to BMI. The A, C, and $\mathrm{E}$ covariance matrices were computed by the product of their respective Cholesky factor-loading matrix and its transpose. The genetic and environmental correlations among the three scales were also derived from the $\mathrm{A}, \mathrm{C}$, and $\mathrm{E}$ variances and covariance. The raw data option in Mx (Neale et al., 2003) was used to conduct model-fitting analyses. To select the best-fitting, most parsimonious model, submodels of the full trivariate Cholesky model were constructed, and the fits of these submodels were compared with the fit of the full model by using the criteria of Akaike information criterion (AIC $=\chi^{2}$ - 2df; Akaike, 1987) and the likelihood-ratio test (LRT).

\section{Results}

\section{Phenotypic Correlations and Twin Correlations}

Table 1 presents phenotypic correlations and MZ and DZ cross-twin, cross-trait correlations for the $\mathrm{CP}$, the $\mathrm{HP}$, and BMI. Although the magnitudes of the phenotypic correlations between BMI and the cold-heat patterns were moderate, they were all significant and consistent with those found in other studies (e.g., Anderson, 1999; Mun et al., 2017; 
TABLE 2

Model-Fitting Results

\begin{tabular}{|c|c|c|c|c|c|c|c|}
\hline & Model description & $-2 \mathrm{LL}$ & $d f$ & $\mathrm{AIC}$ & $\Delta-2 \mathrm{LL}$ & $\Delta d f$ & $p$ \\
\hline 1 & Full model & 13926.3 & 5,231 & 3464.3 & & & \\
\hline 2 & Drop all genetic variances and covariances & 14164.2 & 5,237 & 3690.2 & 237.9 & 6 & $<0.001$ \\
\hline 3 & Drop all shared environmental variances and covariances & 13927.0 & 5,237 & 3453.0 & 0.7 & 6 & 0.99 \\
\hline 4 & $\begin{array}{l}\text { Drop all shared environmental variances and covariances and genetic } \\
\text { covariance between } \mathrm{CP} \text { and HP }\end{array}$ & 13928.1 & 5,238 & 3452.1 & 1.8 & 7 & 0.97 \\
\hline 5 & $\begin{array}{l}\text { Drop all shared environmental variances and covariances and non-shared } \\
\text { environmental covariance between } \mathrm{CP} \text { and } \mathrm{HP}\end{array}$ & 13948.3 & 5,238 & 3472.3 & 22.0 & 7 & $<0.01$ \\
\hline 6 & $\begin{array}{l}\text { Drop all shared environmental variances and covariances and genetic } \\
\text { covariance between HP and BMI }\end{array}$ & 13954.7 & 5,238 & 3478.7 & 28.4 & 7 & $<0.001$ \\
\hline 7 & $\begin{array}{l}\text { Drop all shared environmental variances and covariances and non-shared } \\
\text { environmental covariance between HP and BMI }\end{array}$ & 13928.8 & 5,238 & 3452.8 & 2.5 & 7 & 0.93 \\
\hline 8 & $\begin{array}{l}\text { Drop all shared environmental variances and covariances and genetic } \\
\text { covariance between } \mathrm{CP} \text { and } \mathrm{BMI}\end{array}$ & 13949.0 & 5,238 & 3473.0 & 22.7 & 7 & $<0.01$ \\
\hline 9 & $\begin{array}{l}\text { Drop all shared environmental variances and covariances and non-shared } \\
\text { environmental covariance between } \mathrm{CP} \text { and } \mathrm{BMI}\end{array}$ & 13952.5 & 5,238 & 3476.5 & 26.2 & 7 & $<0.001$ \\
\hline 10 & $\begin{array}{l}\text { Drop all shared environmental variances and covariances, genetic } \\
\text { covariance between CP \& HP, and non-shared environmental } \\
\text { covariance between HP and BMI }\end{array}$ & 13929.9 & 5,239 & 3451.9 & 3.6 & 8 & 0.89 \\
\hline
\end{tabular}

Note: Best-fitting model is indicated in bold type. $\mathrm{LL}=$ log-likelihood. $\mathrm{CP}=$ cold pattern, $\mathrm{HP}=$ heat pattern.

Pham et al., 2016). Negative but low correlation between the $\mathrm{CP}$ and the HP found in the present sample suggested that although some of the symptoms of the two patterns were opposite to each other (e.g., aversion to cold vs. heat), the two patterns could not be considered a single phenotype.

Neither MZ nor DZ cross-twin cross-trait correlation between the CP and the HP was significant. However, MZ cross-twin, cross-trait correlations between the $\mathrm{CP}$ and BMI and between the HP and BMI were significant and greater than the corresponding $\mathrm{DZ}$ cross-twin, cross-trait correlations. None of the DZ cross-twin, cross-trait correlations attained statistical significance. These results suggest that the relationships between the HP and BMI and the $\mathrm{CP}$ and BMI might be due in part to common genes. Note, however, that MZ cross-twin cross-trait correlations were much less than 1.0, suggesting that individual specific environment also plays an important role in the phenotypic relationships.

\section{Model Fitting}

Table 2 presents the results of trivariate Cholesky modelfitting analysis. Model 2 removed genetic variances and covariance from the full model, which resulted in a significant change in chi-square. In contrast, when shared environmental variances and covariance were eliminated from the full model, the difference in chi-square was not significant (model 3). These results suggest that shared environmental factors were not significant for the relationships among the $\mathrm{CP}$, the HP, and BMI. In model 4, we further dropped genetic covariance between the $\mathrm{CP}$ and the HP from model 3, whereas in model 5 we dropped non-shared environmental covariance between the two traits. The resulting chi-square change was significant in model 5 but not in model 4, suggesting that non-shared environmen- tal variance alone explains the relationship between the $\mathrm{CP}$ and the HP. Similarly, models 6 and 7 tested significance of genetic versus non-shared environmental covariance between the HP and BMI. As change in chi-square was significant in model 6 but not in model 7, it was concluded that genetic covariance alone mediated the relationship between the HP and BMI. In model 8, genetic covariance between the $\mathrm{CP}$ and $\mathrm{BMI}$ was removed from model 3 , whereas in model 9, non-shared environmental covariance between the two traits was removed. Both models showed a significant change in chi-square, indicating that both genetic and non-shared environmental covariances are necessary to explain the relationship between the CP and BMI. Model 10 eliminated genetic covariance between the $\mathrm{CP}$ and the $\mathrm{HP}$ and non-shared environmental covariance between the HP and BMI simultaneously from model 3 , which produced a non-significant change in chi-square. From these model comparisons based on the LRT, we concluded that model 10 was the best fit. In agreement with the conclusion from the LRT, AIC was the lowest in model 10 among the models tested.

Figure 1 presents the parameter estimates in the bestfitting trivariate Cholesky model. Additive genetic influences derived from the best-fitting model were 0.38 (95\% CI $[0.31,0.45])$ for the CP, 0.32 (95\% CI $[0.24,0.39])$ for the HP, and 0.84 (95\% CI $[0.82,0.86])$ for BMI. Non-shared environmental influences for corresponding traits were 0.62 (95\% CI $[0.55,0.69]), 0.68$ (95\% CI [0.61, 0.76]), and 0.16 $(95 \%$ CI $[0.14,0.18])$, respectively. These estimates were very close to the estimates found in our previous studies based on univariate biometrical models (Hur et al., 2018).

The best-fitting model also yielded genetic and individual specific environmental correlations, that is, the degree of genetic and environmental overlaps among the three 
TABLE 3

Genetic and Individual Specific Environmental Correlations and Their 95\% Confidence Interval for the Cold Pattern, the Heat Pattern, and BMI Derived from the Best-Fitting Trivariate Cholesky Model

\begin{tabular}{lclc}
\hline & Cold pattern & Heat pattern & BMI \\
\hline Cold pattern & - & $n$ & $-0.22[-0.13,-0.31]$ \\
Heat pattern & $-0.22(-0.28,-0.15)$ & $n$ & $0.31[0.22,0.41]$ \\
BMI & $-0.22(-0.14,-0.30)$ & $n s$ & - \\
\hline
\end{tabular}

Note: Genetic correlations $\left(r_{\mathrm{g}}\right)$ are above the diagonal; Individual-specific environmental correlations $\left(r_{\mathrm{e}}\right)$ are below the diagonal. $95 \% \mathrm{Cl}$ are in square brackets. $n s=$ non-significant.

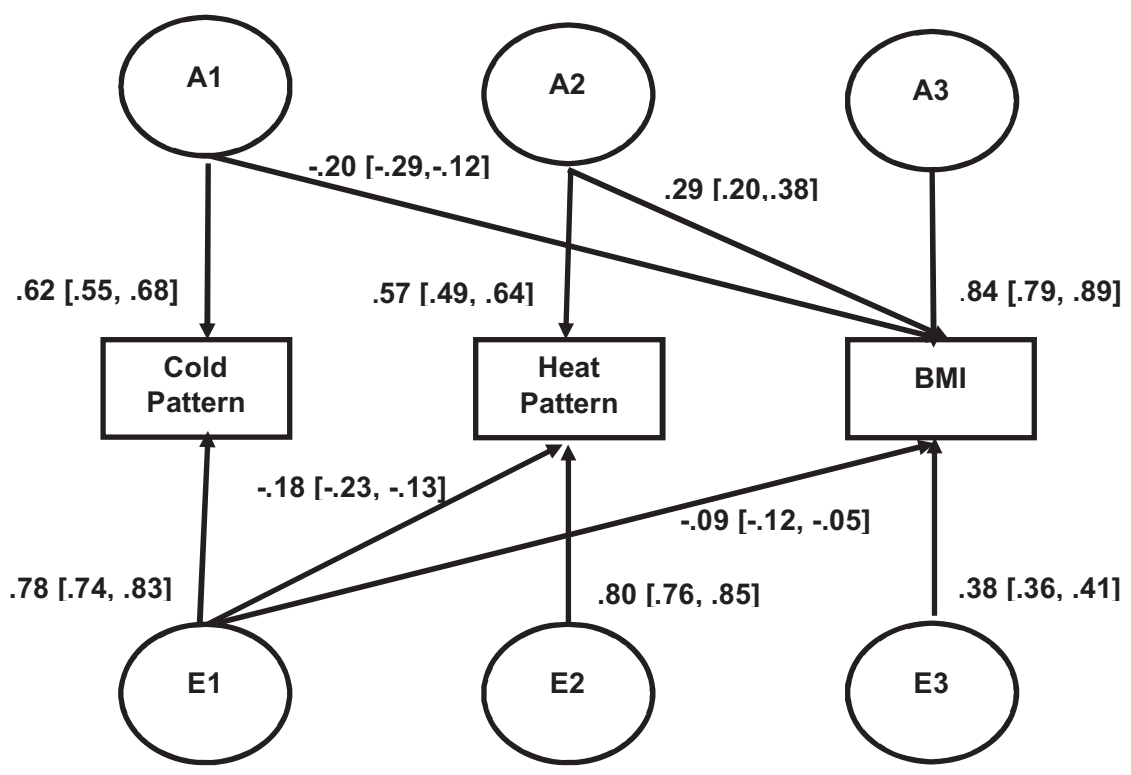

\section{FIGURE 1}

Additive genetic (A1, A2, A3) and individual specific environmental (E1, E2, E3) path coefficients in the best-fitting trivariate Cholesky factor model for cold-heat patterns and BMI. Path estimates should be squared to obtain variance due to each factor. Note: $95 \%$ Cls are in square brackets.

traits (see Table 3$)$. The genetic correlation was $0.31(95 \%$ CI $[0.22,0.41])$ between BMI and the HP, while the individual specific environmental correlation was not significant, suggesting that the phenotypic association between BMI and the HP can be explained entirely by the shared genetic basis. The genetic and individual specific environmental correlations between the CP and BMI were both 0.22 , which suggested that $65 \%$ of the phenotypic correlation between the CP and BMI was attributable to common genes, with the remaining $35 \%$ being due to common individual specific environmental factors. While the genetic correlation between the CP and the HP was not significant, the individual specific environmental correlation was $-0.22(95 \%$ CI $[-0.28,-0.15])$. These results suggested that the $\mathrm{CP}$ and the HP may be genetically independent and that common environmental factors alone can explain the phenotypic correlation between the CP and the HP.

\section{Discussion}

The present study explored shared etiologies of the relationships among cold-heat patterns and BMI. The results showed that the phenotypic relationships of these traits were mediated in part by common genetic influences. That is, many of the genes affecting BMI also influence the HP and the $\mathrm{CP}$, perhaps as a result of some general mechanisms such as the metabolic rate. The metabolic rate has been shown to be a correlate of BMI (Hasson et al., 2011) and heritable (Bouchard et al., 1993), and negatively associated with the CP and positively associated with the HP (Mun et al., 2017; Yoshino et al., 2013).

The genetic correlations were positive between the HP and BMI and negative between the CP and BMI. The signs of these genetic correlations agreed with those of the phenotypic correlations. Thus, our findings suggest that genes that increase the HP can increase BMI, whereas genes that increase the CP can lower BMI. 
Although the phenotypic relationship between the HP and BMI was explained entirely by common genes, the relationship between the $\mathrm{CP}$ and BMI was due in part to the common individual-specific environmental influence as well. As with the genetic correlation, the sign of the individual-specific environmental correlation between the $\mathrm{CP}$ and $\mathrm{BMI}$ was negative, suggesting that certain environmental factors that increase the CP can lower BMI. Candidate environmental factors for this relationship may include smoking and diet.

Interestingly, the present study showed that the HP and the $\mathrm{CP}$ were genetically independent. In support of our findings, Li et al. (2007) in a systems biology study found that immune factors were related to the HP network, while hormones were mainly related to the CP network, suggesting that the biological pathways of the two phenotypes may be largely distinguishable. In a small Norwegian twin sample, Nielsen et al. (2008) examined genetic and environmental relationships between the cold-pressure and the contact heat pain sensitivities, the phenotypes related to the $\mathrm{CP}$ and the HP in our study. The authors also found very modest genetic and environmental overlaps between the two phenotypes. Taken together, these findings imply that information gained from gene identification studies from one pattern may not be generalized to the other pattern, and that separate genetic association studies should be carried out for each pattern.

The present study suggests that although the phenotypic relationship between the $\mathrm{CP}$ and the HP was modest, it was explained mainly by common individual-specific environmental influences. Note, however, the individualspecific environmental correlation between the $\mathrm{CP}$ and the HP contains correlated measurement error as twins in our study completed both the CP and the HP via a telephone interview using a questionnaire with the same Likert scale.

There were a few limitations in the present study. First, we noted that the correlations between BMI and the two patterns were higher in males than in females. However, to increase the sample size, we combined both sexes prior to model-fitting analysis. Future studies should increase sample size and examine whether genetic and environmental etiologies of the relationships between BMI and the two patterns differ across two sex groups. Second, in our study, only the questionnaire method was employed to determine the $\mathrm{CP}$ and the HP. It would be of interest for future studies to use clinician's diagnosis of the $\mathrm{CP}$ and the HP. Finally, our model was based on the assumption that the relationship between the $\mathrm{CP}$ and $\mathrm{BMI}$ is linear as we did not find any non-linearity in our sample. However, some (e.g., Anderson, 1999) have maintained that the CP should be more common in the lowest and the highest groups of BMI. More studies should be carried out to determine the linearity of the relationship between the $\mathrm{CP}$ and BMI.

\section{Acknowledgments}

This work was supported by the 'Development of Health Prediction Technology based on Big Data' (K18092) funded by the Ministry of Science and ICT(MSIT) of Korea given to the Korea Institute of Oriental Medicine (KIOM).

\section{References}

Akaike, H. (1987). Factor analysis and AIC. Psychometrica, 52, 317-332.

Anderson, G. S. (1999). Human morphology and temperature regulation. International Journal of Biometeorology, 43, 99109.

Bae, K.-H., Lee, Y., Park, K.-H., Yoon, Y., Mun, S., \& Lee, S. (2017). Perception of cold and heat pattern identification in diseases: A survey of Korean medicine doctors. Integrative Medicine Research, 6, 26-32.

Bouchard, C., Perusse, L., Deriaz, O., Despres, J. P., \& Tremblay, A. (1993). Genetic influences on energy expenditure in humans. Critical Reviews in Food Science and $\mathrm{Nu}$ trition, 33, 345-350.

Ferreira, A. S., \& Lopes, A. J. (2011). Chinese medicine pattern differentiation and its implications for clinical practice. Chinese Journal of Integrative Medicine, 17, 818-823.

Hasson, R. E., Howe, C. A., Jones, B. L., \& Freedson, P. S. (2011). Accuracy of four resting metabolic rate prediction equations: Effects of sex, body mass index, age, and race/ethnicity. Journal of Science and Medicine in Sport, 14, 344-351.

Hur, Y. M., Kaprio, J., Iacono, W. G., Boomsma, D. I., McGue, M., Silventoinen, K., ... Mitchell, K. (2008). Genetic influences on the difference in variability of height, weight and body mass index between Caucasian and East Asian adolescent twins. International Journal of Obesity, 32, 1455-1467.

Hur, Y.-M., Jeong, H.-U., Chung, K.W., Shin, J. S., \& Song, T.-B. (2013). The South Korean Twin Registry: An update. Twin Research and Human Genetics, 16, 237-240.

Hur, Y.-M., Yu, H., Lee, S., \& Jin, H.-J. (2018). Heritability of cold and heat patterns: A twin study. Twin Research and Human Genetics. 12:1-6. doi:10.1017/thg.2018.17. [Epub ahead of print].

Jian, W.-Y. (2005). Therapeutic wisdom in traditional Chinese medicine: A perspective from modern science. Trends in Pharmacological Sciences, 26, 558-563.

Lee, B. J., Lee, J. C., Nam, J., \& Kim, J. Y. (2018). Prediction of cold and heat patterns using anthropometric measures based on machine learning. Chinese Journal of Integrative Medicine, 24, 16-23.

Li, S., Zhang, Z. Q., Wu, L. J., Zhang, X. G., Li, Y. D., \& Wang, Y. Y. (2007). Understanding ZHENG in traditional Chinese medicine in the context of neuro-endocrine-immune network. IET System Biology, 1, 51-60.

McGue, M., \& Bouchard, T. J. B. (1984). Adjustment of twin data for the effects of age and sex. Behavior Genetics, 14, 325-343.

Mun, S., Kim, S., Bae, K.-H., \& Lee, S. (2017). Cold and spleenqi deficiency patterns in Korean medicine are associated 
with low resting metabolic rate. Evidence-Based Complementary and Alternative Medicine, Article ID 9532073.

Neale, M. C., \& Cardon, L. R. (1992). Methodology for genetic studies of twins and families, London: Kluwer.

Neale, M. C., Boker, S. M., \& Xie, G. (2003). Mx: Statistical modeling (6th ed.). Richmond, VA: Department of Psychiatry, Virginia Commonwealth University.

Nielsen, C. S., Stubhaug, A., Price, D. D., Vassend, O., Czajkowski, N., \& Harris, J. R. (2008). Individual differences in pain sensitivity: Genetic and environmental contributions. Pain, 136, 21-29.

Ooki, S., Yamada, K., \& Asaka, A. (1993). Zygosity diagnosis of twins by questionnaire for twins' mothers. Acta Geneticae Medicae et Gemellologicae, 42, 17-22.

Park, J. H. (2011). Measuring BMI cutoff points of Korean adults using morbidity of BMI-related diseases. Korean Journal of Obesity, 20, 36-43.

Pham, D. D., Lee, J., Kim, G., Song, J., Kim, J., \& Leem, C. H. (2016). Relationship of the cold-heat sensation of the limbs and abdomen with physiological biomarkers. EvidenceBased Complementary and Alternative Medicine, Article ID 27108051.

Qi, Q., Kilpeläinen, T. O., Downer, M. K., Tanaka, T., Smith, C. E., Sluijs, I., .. Qi, L. (2014). FTO genetic vari- ants, dietary intake and body mass index: Insights from 177,330 individuals. Human Molecular Genetics, 23, 69616972.

Silventoinen, K., Jelenkovic, A., Sund, R., Hur, Y. M., Yokoyama, Y., \& Honda, C. (2016). Genetic and environmental effects on body mass index from infancy to the onset of adulthood: An individual-based pooled analysis of 45 twin cohorts participating in the COllaborative project of Development of Anthropometrical measures in Twins (CODATwins) study. American Journal of Clinical Nutrition, 104, 371-379.

WHO Regional Office for the Western Pacific. (2007). WHO international standard terminologies on traditional medicine in the western pacific region. Geneva, Switzerland: World Health Organization.

Yeo, M., Park, K., Bae, K., Jang, E., \& Lee, Y. (2016). Development on the questionnaire of cold-heat pattern identification based on usual symptoms for health promotion - Focused on reliability study. Journal of Physiology \& Pathology in Korean Medicine, 30, 116-123.

Yoshino, T., Katayama, K., Munakata, K., Horiba, Y., Yamaguchi, R., Imoto, S., ... Watanabe, K. (2013). Statistical analysis of hie (cold sensation) and hiesho (cold disorder) in Kampo clinic. Evidence-Based Complementary and Alternative Medicine, Article ID 398458. 